\title{
MOTIVOS GRAVES DE ORDEN PÚBLICO, UNA EXCEPCIÓN A LA PROTECCIÓN REFORZADA CONTRA LA EXPULSIÓN DE CIUDADANOS COMUNITARIOS CON 10 AÑOS DE RESIDENCIA EN EL ESTADO DE ACOGIDA
}

\author{
ISABEL $M^{\mathrm{a}}$ VILLAR FUENTES
}

Profesora de Derecho Procesal en la Universidad de Cádiz

isabel.villar@uca.es

RESUMEN: El estudio analiza el art. 28.3 de la Directiva 2004/38, donde se plantea una protección reforzada, para los ciudadanos comunitarios con más de 10 años de residencia en el Estado comunitario de acogida, contra la expulsión. En concreto se indaga sobre la excepción a esta protección, excepción que se contempla en caso de "motivos imperiosos de seguridad pública", qué alcance tienen estos motivos y en definitiva si los abusos sexuales a una menor pueden ser considerados como tal.

PALABRAS CLAVE: Ciudadanía europea, derecho libre circulación, expulsión, motivos imperiosos de seguridad pública, orden público.

PUBLIC SERIOUS OF GROUNDS OF AN EXCEPTION TO THE REINFORCED PROTECTION AGAINST REMOVAL OF EU CITIZENS 10 YEARS OF RESIDENCE IN THE STATE OF WELCOME

ABSTRACT: This study analyzes the art. 28.3 of Directive 2004/38, which lays greater protection for EU citizens which complete over 10 years of residence in the host community, against removal. Specifically it investigates the exception to this protection, exception provided in case of "imperative grounds of public security", what is the scope of these reasons and ultimately whether sexual abuse to a minor can be considered as such.

KEYWORDS: European citizenship, free movement rights, removal, imperative grounds of public security, public order. 


\section{INTRODUCCIÓN}

El Tribunal de Justicia de la Unión Europea se pronuncia en la decisión prejudicial planteada por el Oberverwaltungsgerischt für das Land Nordrhein-Westfalen (Alemania) sobre la interpretación del concepto "motivos imperiosos de seguridad pública" del art. 28, apartado 3 de la Directiva 2004/38/CE, para poder llevar a cabo la expulsión de un ciudadano extranjero de un Estado miembro, en el que ha residido más de diez años. De modo más concreto pregunta si los "motivos imperiosos de seguridad pública" implica "solamente los riesgos para la seguridad interior y exterior del Estado, entendida como la permanencia del Estado con sus instituciones y sus servicios públicos fundamentales, la supervivencia de la población, las relaciones exteriores y la convivencia pacífica de los pueblos”.

Esta consulta se ubica en los siguientes hechos: el Sr. Infusino es ciudadano italiano nace el 3 de septiembre de 1965 y vive en Alemania desde 1987. Este ciudadano no tenía ninguna formación escolar, ni empleo estable, solo había llevado a cabo trabajos con carácter temporal. Entre los años 1990 y 2001 obligó a la hija de su pareja, desde los ocho años a mantener relaciones sexuales con él, bajo la amenaza de matar a su madre o a su hermano. Por estos hechos fue condenado por el Tribunal Regional de Colonia el 16 de Mayo de 2006, a una pena privativa de libertad de 7 años y seis meses. Pena que cumpliría el 9 de julio de 2013. Pero el Oberbürgermeisterin der Stadt Remschid en resolución de 6 de Mayo de 2008, tras ser desestimado el Recurso del Sr. Infusino, declaró la pérdida de la residencia y ordenó la ejecución inmediata, caso contrario sería expulsado a Italia.

De modo que la consulta del alto tribunal alemán es aclarar si los "motivos imperiosos de seguridad pública”, que plantean una protección reforzada contra la expulsión, se ciñen exclusivamente a riesgos para la seguridad interior y exterior del Estado. El alcance detallado de consulta alcanza a si esos motivos se circunscriben a 
supervivencia de la población, relaciones exteriores...aquello que pueda poner en peligro la estabilidad, seguridad, pervivencia del Estado, tanto en su proyección exterior, como en lo relativo a personas e instituciones nacionales. Estas cuestiones intentan aclararse también a la luz de la ST Tsakourdis que más adelante será analizada.

Para poder aplicar la limitación a los derechos de libre circulación y libre residencia a un ciudadano europeo es preciso, por tanto que se lleve a cabo debido a razones de "orden público, seguridad pública o salud pública", nunca por tanto debido a razones económicas. Caso de que el ciudadano europeo tenga residencia permanente, o en el caso máximo de protección, la residencia supere los diez años, será preciso que se trate de motivos "graves" o "imperiosos", respectivamente de seguridad pública. La citada Directiva para poder valorar "seguridad pública" y "orden público" acude al principio de proporcionalidad y a la necesidad de que se trate de una conducta personal, así como a evaluar los lazos económicos y sociales, en definitiva la raigambre con el Estado de acogida.

Siendo ésta la cuestión, el contenido de los "motivos imperiosos de seguridad pública", planteada por el tribunal alemán al Tribunal de Justicia de la Unión Europea, surge otro punto de análisis, si el ciudadano italiano puede invocar la protección reforzada, por larga duración, del art 28.3 de la Directiva 2004/38. Ambos interrogantes son analizados en este comentario.

\section{CIUDADANÍA Y DERECHO LIBRE CIRCULACIÓN}

Antes de entrar en los límites a los derechos de libre circulación y residencia, debemos tener en cuenta el por qué de estos derechos, así como la preocupación del legislador comunitario por protegerlos hasta las últimas consecuencias. 
El derecho a la ciudadanía europea supuso un grupo diferenciado y reconocido de derechos en la Carta de Derechos Fundamentales de Niza, donde una de las categorías que recogía eran los derechos exclusivos para los ciudadanos europeos ${ }^{1}$. El concepto de ciudadanía europea existe en el Tratado de Funcionamiento de la Unión Europea (art. 21) y en la Carta de los Derechos Fundamentales de la Unión Europea (art. 45).

La ciudadanía europea implica un reconocimiento jurídico a los nacionales de los Estados miembros, en el resto de los Estados de la Unión Europea. De tal modo que se crea una categoría distinta y más favorable que la de "extranjero" y lógicamente diferenciada también de la de "nacional" de cada Estado miembro. Por tanto se puede afirmar que es un grupo de derechos "especiales" para los ciudadanos europeos, los que a su vez conforman el concepto de ciudadanía, entre otros cabe destacar: derecho a entrar y salir del territorio, derecho a ejercer una profesión... Pero el rasgo diferenciador de la ciudadanía europea lo conforma el derecho de libre circulación y residencia en el territorio de los Estados miembros. La libre circulación de personas tiene dos concepciones: "la libertad de circulación en el territorio de un Estado como derecho fundamental, y la libre circulación entre Estados como fruto de procesos de integración económica"2. La fundamentación jurídica de la ciudadanía europea no encuentra respaldo en cuestiones económicas o laborales, sino en una libertad política ${ }^{3}$.

\footnotetext{
${ }^{1}$ MANGAS MARTín, A., Carta de los derechos Fundamentales de la Unión Europea, Comentario artículo por artículo, Bilbao 2008, pag. 57. Diferencia los tres grupos de derechos en los que se iba a articular la Carta de Niza: derechos de libertad e igualdad y derechos procesales fundamentales, derechos de la ciudadanía europea, reservados a los ciudadanos europeos, y los derechos económicos y sociales.

${ }^{2}$ IGLESIA SÁNCHEZ, S., La libre circulación de los extranjeros en la Unión Europea: El régimen de movilidad en las Directivas de la UE en materia de inmigración, Madrid 2010, pags. 353 y ss: “...la libre circulación de personas como un elemento del proceso de integración económica supone la base sobre la cual se sustenta la emergencia de la concepción de la libre circulación de personas como una libertad fundamental, más allá de su contenido puramente económico.”.

3 MANGAS MARTÍN, A; Carta de los Derechos fundamentales de la Unión Europea...,op. cit., pags. 720 y ss, "El salto cualitativo se dio en el Tratado de Maastrich al configurar la institución de la ciudadanía de la Unión y su estatuto de derechos específicos, entre los que figura el derecho de libre circulación y residencia de todo ciudadano de la Unión. La vigente normativa de desarrollo del derecho ha superado el enfoque sectorial y económico del derecho de libre circulación y residencia limitado hasta 1993 para los agentes económicos”.
} 
Se trata éste, de un derecho público subjetivo alegable y objeto de protección ante los Tribunales ${ }^{4}$. Este derecho de libre circulación y residencia de los ciudadanos europeos se garantiza y proclama de una forma absoluta, sin embargo ello no es óbice para que el propio Derecho comunitario regule ciertas condiciones y límites, en lo concerniente a las actividades económicas o profesiones que se desarrollen, así como "restricciones".

El ejercicio del derecho de libre circulación y residencia de los ciudadanos europeos ya desarrollado por la Directiva 64/221 del Consejo, de 25 de febrero de 1964, tienen un especial tratamiento en la Directiva 2004/38 del Consejo, de 29 de Abril de 2004. Esta Directiva que deroga las anteriores en esta materia, al desarrollar el derecho de residencia y el de residencia permanente (art 22), establece que tales residencias, no podrán tener límites distintos a los que se previeran para los nacionales ${ }^{5}$. Siguiendo en esa línea la Directiva en el art. 28 plantea una protección contra la expulsión y de este modo es denominado por la Directiva 2004/38. Directiva destinada a los ciudadanos de la $\mathrm{UE}^{6}$, ciudadanía comunitaria, que superan el concepto de extranjero y lo "aproxima" al nacional. Partiendo del concepto de ciudadano europeo plantea la posible decisión de expulsión por razones de orden público, con la siguiente advertencia: “...el Estado miembro deberá tener en cuenta, en particular, la duración de la residencia del interesado en su territorio, su edad, estado de salud, situación familiar y económica, su

\footnotetext{
${ }^{4}$ MANGAS MARTíN, A., Carta de los Derechos Fundamentales..., op. cit., pag. 723. Advierte de la existencia de este derecho de libre circulación y residencia, con independencia de su actividad económica y de la pertenencia al grupo familiar.

${ }^{5}$ MANGAS MARTín, A., Carta de los Derechos fundamentales..., op. cit., pag. 359, cita el punto 1.7 de la Exposición de Motivos de la Propuesta de Directiva sobre el estatuto de los nacionales de terceros países residentes de larga duración: "que la circulación de los ciudadanos de la Unión entre los Estado miembros, mutatis mutandi, en condiciones similares, si no iguales, a las de los ciudadanos de un Estado miembro que circulan o cambian de residencia o actividad dentro de su propio país:".

${ }^{6}$ MANGAS MARTÍN, A; Carta de los Derechos fundamentales..., op. cit., pag. 358. "La ciudadanía de la Unión elimina; al menos de hecho y en buena medida de derecho, la categoría jurídica de extranjero referida a un nacional de un Estado miembro;...Así pues la esfera de derechos y obligaciones del ciudadano de la Unión fuera del Estado del que es nacional coincide en gran medida con la esfera nacional como consecuencia de la aplicación del derecho de no discriminación al ejercicio del derecho de libre circulación y residencia.”.
} 
integración social y cultural en el Estado miembro de acogida y la importancia de los vínculos con su país de origen.”. El apartado 1 del art. 28 de la Directiva 2004/38 recoge el arraigo personal, familiar, laboral del ciudadano europeo, así como razones que podemos calificar de humanitarias, como: edad, salud etc, a tener en cuenta, antes de tomar la decisión de expulsión. Pero el siguiente apartado ciñe la expulsión a los ya mencionados motivos graves de orden público. De este modo está excluyendo de forma tácita la expulsión por razones económicas o laborales.

\section{LIMITACIONES AL DERECHO DE RESIDENCIA.}

Sin embargo el objeto de estudio de esta sentencia, se centra en la existencia de limitaciones tanto al derecho de entrada como al de residencia, recogidas el Capítulo VI Limitaciones del derecho de entrada y del derecho de residencia por razones de orden público, seguridad pública o salud pública. Podemos afirmar que se trata de unas excepciones al derecho de residencia de los ciudadanos europeos en los Estados miembros, excepciones que para poder entrar en juego, precisan de unas premisas. El art. 27 del citado Capítulo de la Directiva 2004/38/CE contiene los Principios generales, donde permite a los Estados limitar estos derechos, por razones de orden público, seguridad pública y salud pública y nunca por razones económicas.

La Directiva 2004/38 en el Capítulo VI podríamos afirmar que distingue dos clases de limitaciones al derecho de entrada y de residencia: por una parte los arts 27, 28 y 29 , amparadas en razones de seguridad y orden público (con distintas intensidades) y por otra el art. 33, donde reconoce la posibilidad y por tanto la libertad de los Estados miembros de aplicar como pena o medida accesoria la orden de expulsión del territorio. En este último supuesto queda condicionado a los requisitos de los arts. 27, 28 y 29.

\footnotetext{
${ }^{7}$ Las limitaciones de orden público, seguridad pública y salud pública se encuentran también en materia de inmigración, admisión de estudiantes, investigadores y trabajadores altamente cualificados.
} 
De acuerdo con la discrecionalidad reconocida por la Directiva $^{8}$, la normativa nacional alemana Ley sobre Circulación de los Ciudadanos de la Unión de 30 de Julio de 2004, en su art. 6 prevé la posibilidad de perder el derecho de residencia por razones de orden público, seguridad y salud pública. Sin embargo advierte que "una condena penal no bastará por si sola para justificar" la decisión de la expulsión. La Ley alemana condiciona la consideración de una condena penal, al examen de la actualidad y realidad de la amenaza para el orden público ${ }^{9}$. Esta condición del art. 6 de la Ley nacional, coincide con la que recoge en el art. 33 de la Directiva 2004/38, al plantear la modalidad de expulsión, antes mencionada, como medida accesoria ${ }^{10}$. Esta Ley añade que en el caso de residencia de más de diez años en el territorio de la República federal solo podrá adoptarse la expulsión por "razones imperiosas de seguridad pública".

Pero la Directiva al regular estos límites, como supuestos de expulsión por razones de orden público (art. 27.1, Directiva 2004/38), así como los criterios a tener en cuenta antes de poder aplicarla (art. 27.2 y art. 28.1, Directiva 2004/38), incluye una protección reforzada ante la expulsión en el caso de residencia superior a diez años, en cuyo caso deberá sustentarse en "motivos imperiosos de seguridad pública" (art. 28.3, Directiva 2004/38).

A) "Motivos imperiosos de seguridad pública"

\footnotetext{
${ }^{8}$ IGLESIA SÁNCHEZ, S., La libre circulación de los extranjeros..., op. cit., pag. 263: “...los Estados miembros no están limitados de igual modo que en relación con los ciudadanos de la Unión a la hora de tomar en cuenta los antecedentes penales.".

${ }^{9}$ La sentencia de 24 de Noviembre de 2008 del Verwaltungsgericht Stuttgart, ante la impugnación del Sr. Tsakourdis por la expulsión del territorio alemán por la condena por tráfico de estupefacientes, señala: "Una condena penal no es suficiente por si sola para fundar la pérdida del derecho de entrada y de residencia de un ciudadano de la Unión, pérdida que exige amenaza efectiva y suficientemente grave; que afecte a un interés fundamental de la sociedad, en el sentido del artículo 6, apartado 2, de la FreizüG/EU.”.

${ }^{10}$ El art.33.2 de la Directiva 2004/38 advierte que si se ejecuta dicha orden dos años después de haberse dictado, deberá llevarse a cabo una comprobación de la actualidad y realidad de la amenaza para el orden público.
} 
La consulta planteada por el Oberverwaltungsgeritcht für das Land NordrheinWestfalen al Tribunal de Justicia de la Unión Europea, es la necesidad de interpretación por parte de éste del art. 28. Apartado 3, letra a) de la Directiva 2004/38/CE del Parlamento Europeo y del Consejo, de 29 de Abril de 2004, relativo al derecho de los ciudadano de la Unión y de los miembros de sus familias a residir libremente en el territorio de los Estados miembros se centra en lo siguiente. Conocer si "motivos imperiosos de seguridad pública", afecta sólo a los riegos para la seguridad interna y externa del Estado, entendida como la permanencia del Estado con sus instituciones y sus servicios públicos fundamentales, la supervivencia de la población, las relaciones exteriores y la convivencia pacífica de los pueblos.

El Tribunal de Justicia de la Unión Europea se encuentra en la necesidad de interpretar el concepto de "motivo grave de orden público y seguridad pública". Es más tiene que llenar de contenido y poner límites a unos motivos que quedan recogidos en la Directiva 2004/38 de una forma difusa. La Directiva, que estamos tratando plantea una especie de graduación del concepto de "seguridad pública", pues habla de "orden público y seguridad pública" (art. 27.1) a la hora de limitar la libre circulación y residencia de un ciudadano de la Unión; "motivos graves de orden público o seguridad pública" (art. 28.2) para tomar la decisión de la expulsión de un ciudadano de la UE con derecho de residencia permanente; y "motivos imperiosos de seguridad pública"11 (art. 28.3) si la residencia del ciudadano que se pretende expulsar es superior a diez años. Este último supuesto refleja, como venimos insistiendo en una excepcionalidad y una gravedad necesaria, para poder aplicar la orden de expulsión a un ciudadano europeo con residencia en un Estado miembro superior a los diez años.

\footnotetext{
11 La Sentencia del Tribunal de Justicia de la Unión Europea, de 23 de Noviembre de 2010, (ST Taskouridis), en el apartado "Sobre las Cuestiones Prejudiciales", distingue: "motivos imperiosos de seguridad pública, concepto que es considerablemente más limitado que el de motivos graves, en el sentido del aparatado 2 de este artículo, el legislador de la Unión ha pretendido claramente circunscribir las medidas fundadas en dicho apartado 3 a circunstancias excepcionales...".
} 
La "seguridad pública" y el "orden público" en distintas ocasiones es interpretado como aquellos ataques a la seguridad del Estado, a la paz interior y exterior o a los valores democráticos. En este punto es interesante la distinción que hace el Verwaltungsgericht Stuttgart ${ }^{12}$ entre "seguridad pública" y "orden público", de modo que, la primera compromete la seguridad interior y exterior de un Estado y la segunda al orden penal interno. Afirmación que hace fundamentándose en la decisiva Sentencia del Tribunal de Justicia de la Unión Europea, de 23 de Noviembre de 2010, (ST Tsakouridis). Hay que tener en cuenta, que la propia Directiva alega la independencia de cada Estado miembro para definir y concretar el alcance de "seguridad pública". La propia consulta de la cuestión prejudicial al Tribunal de Justicia de la Unión Europea, plantea la necesidad de concreción y contenido de los "motivos imperiosos de la seguridad pública" a la luz de la sentencia de 23 de noviembre de 2010, Tsakouridis. La ST Tsakouridis efectivamente analiza el concepto de "motivos imperiosos de seguridad pública”, que pueden justificar una medida de expulsión de un ciudadano de la Unión, sin embargo resulta difícil de extrapolar con el caso que nos ocupa, pues se trata de tráfico de estupefacientes. Declara el Tribunal de Justicia en la ST Tsakouridis: "la lucha contra la criminalidad asociada al tráfico de estupefacientes mediante banda organizada puede estar comprendida en el concepto de motivos imperiosos de seguridad pública que pueden justificar una medida de expulsión de un ciudadano de la Unión”. La diferencia entre la criminalidad asociada a banda organizada con un despliegue de medios económicos y humanos para delinquir, está claro que difiere del ilícito penal del Sr. Infusino ${ }^{13}$. Partiendo de la base de la gravedad del delito de agresión

\footnotetext{
${ }^{12}$ La Sentencia del Tribunal de Justicia de la Unión Europea, de 23 de Noviembre de 2010 (ST Tsakourdis), recoge en el apartado "Litigio Principal y Cuestiones Prejudiciales": "El Verwaltungsgericht Stuttgart declaró que no existían "motivos imperiosos de seguridad pública", en el sentido del art. 6, a partdo 5, última frase, FreizügG-EU, que justificasen una medida de expulsión. La seguridad pública solo comprende la seguridad interior y exterior de un Estado miembro y, por lo tanto, es un concepto más limitado que el de orden público, que comprende así mismo el orden penal interno. El hecho de que se haya superado la pena mínima mencionada en el art 6, apartado5, última frase, de la FreizügG-EU, no permite llegar a la conclusión de que existen motivos imperiosos de seguridad pública a los efectos de una medida de expulsión. A juicio del Verwaltungsgericht Stuttgart, el Sr. Tsakouridis tal vez pueda representar un peligro importante para el orden público, pero de ningún modo para la existencia del Estado y de sus instituciones ni para la supervivencia de la población.".

13 Conclusiones del Abogado General, Sr. Yves Bot en el asunto C-348/09 (Sr. Infusino contra Oberbürgermeisterin der Stadt Rermscheid), apartado "Mi análisis", pto. 26: reconoce que el tráfico de
} 
sexual y violación a una menor, de manera continuada, no parece que puede calificarse como ataque a la seguridad pública ("motivo imperioso contra la seguridad pública"). El Fiscal General, el Sr. Yves Bot en las Conclusiones del asunto C-348/09, objeto de esta sentencia, reconoce el grave daño que infringe a la sociedad pero no lo considera agresión a la "seguridad pública" del art. 28.3 de la directiva 2004/38 .

La Directiva 2004/38 en los Principios Generales del art. 27, al referir estas razones de orden público o seguridad jurídica, señala dos parámetros importantes para ser valoradas: "proporcionalidad" y "conducta personal del interesado" y nunca alegadas con "fines económicos".

\section{Proporcionalidad}

La "proporcionalidad" exigida a la hora de adoptar las medidas por razones de orden público o seguridad pública es tenida en cuenta en otras sentencias del Tribunal de Justicia de la Unión Europea, debido a la gravedad de la medida de expulsión. Ésto se debe a que "constituye una medida que puede perjudicar seriamente a las personas, que haciendo uso de los derechos y libertades conferidas por el tratado (CE), se integraron voluntariamente en el Estado miembro de acogida. Conviene por lo tanto limitar el alcance de estas medidas de conformidad con el principio de proporcionalidad para tener en cuenta el grado de integración de las personas en cuestión, la duración de la residencia en el Estado miembro de acogida, su edad...” ${ }^{15}$. En este caso el Tribunal de Justicia de la Unión Europea aplica el criterio de proporcionalidad de la Directiva,

estupefacientes a través de banda organizada supone una "criminalidad difusa, dotada de medios económicos y operativos impresionantes y que tiene con mucha frecuencia conexiones transnacionales".

14 Conclusiones del Abogado General, Sr. Yves Bot en el asunto C-348/09 (Sr. Infusino contra Oberbürgermeisterin der Stadt Rermscheid), apartado "Mi análisis", pto. 28: "Aunque es innegable que el abuso sexual de un menor de 14 años, la agresión sexual y la violación constituye un ataque especialmente grave a un valor fundamental de la sociedad, no considero que esta clase de actos estén comprendidos en el concepto de "seguridad pública", en el sentido del artículo 28, apartado 3, de la Directiva 2004/38."

${ }^{15}$ Sentencia del Tribunal de Justicia, de 23 de Noviembre de 2010, (ST Tsakouridis): "Cuanto mayor sea la integración de los ciudadanos de la Unión Europea y de los miembros de su familia en el Estado miembro de acogida, tanto mayor debería ser la protección contra la expulsión...”. 
respecto a las circunstancias e indicios de arraigo del ciudadano con el Estado miembro de acogida, a la hora de plantear una expulsión.

Estas circunstancias, llamémosles personales, son las que establece la Directiva 2004/38 en el art. 28.1, al tratar la defensa del derecho de libre circulación y residencia, ante la posible decisión de expulsión, en virtud de razones de orden público o seguridad pública. Tiene en cuenta la necesidad de atender el Estado miembro de acogida, a la duración de la residencia del interesado, edad, estado de salud, situación familiar y económica, integración social y cultural en el Estado de acogida (art. 28.1, Directiva 2004/38). De todas estas circunstancias a tener en cuenta, en el caso que nos ocupa, solo la duración de la residencia, resulta favorable al Sr. Infusino. El Sr. Infusino nació en Italia y obtuvo la primera tarjeta de residencia en Alemania en 1987, fue condenado por delito de abusos sexuales, agresión sexual y violación de una menor en mayo de 2006 y perdió el derecho de entrada y residencia en el territorio alemán, mediante resolución de mayo de 2008.

En la sentencia estudiada, la residencia del ciudadano italiano efectivamente es prolongada, sin embargo su integración social y económica en Alemania, queda en entredicho, pues la sentencia del Tribunal de Justicia de la Unión Europea recoge que el sujeto: "está soltero y no tiene hijos. No ha completado ninguna formación escolar y sólo ha trabajado en Alemania con carácter temporal.". Respecto a la escasa integración en la sociedad y la duración de su residencia, es interesante la reflexión que hace el Sr. Yves Bot en sus Conclusiones como Abogado General: “...su comportamiento y la forma de de comisión de los actos demuestran que en realidad no se ha integrado efectivamente y que por tanto no puede disfrutar de la protecciónreforzada.”. La propia sentencia en el punto 12 reconoce la falta de integración social y económica del Sr. Infusino en la sociedad alemana.

Pero en otras ocasiones el Tribunal de Justicia de la Unión Europea acude a la proporcionalidad, para valorar la gravedad de los hechos. Es el caso de la Sentencia 
Calfa $^{16}$, donde el Tribunal de Justicia de la Unión Europea aplica el principio de proporcionalidad entre la medida de expulsión (en esta caso expulsión de por vida) y la gravedad de las infracciones.

$\mathrm{Si}$ analizamos la gravedad de la conducta criminal en la sentencia que nos ocupa debemos tener en cuenta la calificación de los hechos por el artículo 3 de la Directiva 2011/93/UE "Infracciones relacionadas con los abusos sexuales”, en su aparatado 4 establece: "Realizar actos de carácter sexual con un menor que no ha alcanzado la edad de consentimiento sexual se castigará con penas privativas de libertad de una duración máxima de al menos cinco años.” y como agravante en el artículo 9, apartado b): “b) que la infracción haya sido cometida por un miembro de la familia, una persona que convivía con el menor o una persona que haya abusado de su posición reconocida de confianza o de autoridad". Agravante que concurre en los hechos del Sr. Infusino, pues se produjeron con la hija de su pareja, con la cual convivía. No cabe duda que las actuaciones por los que fue condenado el Sr. Infusino, son calificados como graves, tanto por el ilícito objetivamente analizado, como por la víctima, en esta caso una menor $^{17}$. Pero se trata de un delito que se sitúa en el contexto familiar, por tanto sin una repercusión en la seguridad pública ${ }^{18}$.

La proporcionalidad es proclamada también como principio para valorar si existen medios menos lesivos para evitar los daños a la seguridad pública. De este modo es

\footnotetext{
${ }^{16}$ Sentencia del Tribunal de Justicia de la Unión Europea, de 19 de Enero de 1999. La medida se decreta con motivo la condena por tenencia y consumo de estupefacientes de una ciudadana italiana en Grecia.

${ }^{17}$ La Directiva 2011/93 señala la especial gravedad, importancia y necesidad de protección de los menores ante cualquier situación de abuso o explotación sexual: "Los abusos sexuales y la explotación sexual de los menores, incluida la pornografía infantil, constituyen graves violaciones de los derechos fundamentales y, en particular, de los derechos del niño a la protección y los cuidados necesarios para su bienestar, tal como establecen la Convención de las Naciones Unidas sobre los Derechos del Niño de 1989 y la Carta de los Derechos Fundamentales de la Unión Europea".

18 Conclusiones del Abogado General, Sr. Yves Bot en el asunto C-348/09 (Sr. Infusino contra Oberbürgermeisterin der Stadt Rermscheid), apartado "Mi análisis", pto. 44: "desde el punto de vista criminológico que ese tipo de conducta, situada en un contexto exclusivamente familiar, no puede equipararse a la de los "depredadores sexuales", aunque el Sr. I. constituye innegablemente un peligro en la esfera familiar, no está acreditado por la naturaleza de los actos cometidos que constituya una amenaza para la seguridad de los ciudadanos de la Unión...”.
} 
citada por el Fiscal General Sr. Yves Bot en las Conclusiones del caso Tsakouridis "proceder al test de proporcionalidad, teniendo presente que dicha medida sólo puede justificarse por esos motivos si es necesaria para la protección de los intereses que pretende garantizar, siempre que esos objetivos no puedan alcanzarse con medidas menos restrictivas. ${ }^{\prime 19}$.

2. Conducta personal del interesado

La necesidad de aplicar las medidas limitativas al derecho de residencia por razones de orden público, solo fundamentada en la conducta del interesado, ya lo recogía la anterior Directiva 64/221, de igual modo que lo hace la Directiva 2004/38 en al art. 27.2. Esto excluye la aplicación de la expulsión a la mera pertenencia de organizaciones o asociaciones ${ }^{20}$. El objeto de estudio no plantea, en este punto, dudas. Dada la conducta juzgada y por tanto los delitos cometidos, se trata de una actuación personal y directa del Sr. Infusino.

B) Derecho a la protección reforzada

La cuestión del derecho a la específica protección reforzada del art 28.3 del Directiva 2004/38, supone reconocer que la misma no se produce de forma automática por el simple hecho formal del transcurso del tiempo. Los diez años de residencia del ciudadano europeo en el Estado miembro no son un mero cómputo, sino que implican que las sucesivas renovaciones de residencia y más aún el alcanzar la residencia permanente, se producen debido a una convivencia social y pacífica.

\footnotetext{
${ }^{19}$ Conclusiones Fiscal General Sr. Yves Bot, en el caso C-145/09, (caso Tsakouridis), pto. 85, cita las sentencias Boscher, Omega y Oteiza Olazabal, donde el Tribunal de Justicia de la Unión Europea, construye el principio de proporcionalidad, como medio de comprobación de inexistencia de decisión menos lesiva que la expulsión.

${ }^{20}$ Sentencia del Tribunal de Justicia de la Unión Europea, de 4 de Diciembre de 1974, para que se pronuncie sobre la negativa de entrada de una ciudadana holandesa en el Reino Unido, amparándose en pertenencia a la Iglesia de la Cienciología. Negativa que, en un principio chocaría con el presupuesto para la privación de permiso de residencia: "las medidas de orden público o de seguridad pública, deberán ser fundamentadas exclusivamente en el comportamiento personal del individuo a que se aplique.". A este respecto el Tribunal de Justicia de la Unión Europea concreta: “...los Estados miembros están obligados a no tener en cuenta factores distintos del comportamiento personal, la seguridad jurídica de los afectados exige que pueden alegar esta obligación...”.
} 
Los hechos que dan lugar a la condena se prolongan a lo largo de 11 años, desde 1990 hasta 2001 y no parecen éstos un ejemplo de integración, pues se trata de una actividad ilícita para cualquier Estado. Alegar una residencia prolongada en el Estado de acogida, mientras se están llevando a cabo conductas penales en dicho Estado, no deja de sugerir una invocación fraudulenta. La razón por la cual se pudieron renovar tales permisos fue que no descubrió la execrable actividad criminal, que estaba llevando a cabo el Sr. Infusino en territorio alemán.

El derecho a la protección reforzada debe ser analizado antes que la existencia de "motivos imperiosos de seguridad pública", primero debe darse la condición del beneficio, del derecho. En las Conclusiones del Sr. Yves Bot en el caso Tsakouridis, éste, el Fiscal General cuestiona el asunto de las interrupciones en el tiempo de la residencia de Tsakouridis. La duda se produce en torno a si tales lapsus de tiempo, pudieron producir una interrupción de los diez años de residencia requeridos, para disfrutar de la especial protección ${ }^{21}$.

En el asunto del Sr. Infusino la posición del Fiscal General en sus Conclusiones, así como la del Gobierno Holandés es totalmente contraria al reconocimiento de la protección reforzada “... ya que su comportamiento y la forma de comisión de los actos demuestran que en realidad no se ha integrado efectivamente..." 22.

\footnotetext{
${ }^{21}$ Conclusiones Fiscal General Sr. Yves Bot, en el caso C-145/09, (caso Tsakouridis), en el aparatado V. Conclusión: "Las ausencias t5ewmporales que no desvirtúen el vínculo estrecho que une al ciudadano de la Unión con el Estado de acogida, circunstancia que corresponde comprobar al juez nacional, no tienen incidencia en el cómputo de plazo exigido por el artículo 28, apartado 3, letra a), de la Directiva 2004/38.".

${ }^{22}$ Conclusiones Fiscal General Sr. Yves Bot, en el caso C-348/09, (caso Sr. Infusino), en los ptos. 49 y 50, apoya su oposición y la del gobierno holandés en el propio Tribunal de Justicia en el asunto Kol donde afirmaba que "un comportamiento fraudulento podía privar a su autor del beneficio de una autorización de residencia.”, cita al tribunal de Justicia en el asunto Kol: "debe excluirse que el ejercicio de un empleo al amparo de una autorización de residencia expedida a raíz de un comportamiento fraudulento que dio lugar a una condena pueda generar derechos a favor de un trabajador turco o justificar una confianza legítima en éste....”.
} 
El Sr. Yves Bot en sus Conclusiones lleva a cabo una exposición pormenorizada del uso fraudulento del tiempo de residencia, de modo que tal y como señalábamos anteriormente, que no se puede tratar de una mera cuestión temporal, de transcurso del tiempo, sino que implica una cuestión de "calidad" de ese tiempo. En definitiva en el espíritu de la Directiva 2004/48setá el hecho de que a mayor tiempo de residencia, mayor grado de arraigo y de identificación del ciudadano europeo en el Estado miembro, extremos éstos que no encajan con la actividad criminal del ciudadano italiano. La Conclusión del Sr. Yves Bot es que "un ciudadano de la Unión no puede invocar el derecho a una protección reforzada contra la expulsión en virtud de esa disposición cuando está demostrado que este ciudadano fundamenta ese derecho en un comportamiento infractor constitutivo de una perturbación grave del orden público del Estado miembro.”.

\section{CONCLUSIÓN}

A la vista de la jurisprudencia del Tribunal de Justicia de la Unión Europea los delitos cometidos por el Sr. Infusino no deberían ser calificados como "motivos imperiosos de seguridad pública”, y en ese sentido se pronuncia el Fiscal General del caso, sin embargo en este caso el Tribunal de Justicia resuelve: "que infracciones penales como las mencionadas en el artículo 83 TFUE, apartado 1, párrafo segundo, constituyen un menoscabo especialmente grave de un interés fundamental de la sociedad capaz de representar una amenaza directa para la tranquilidad y la seguridad física de la población y que por consiguiente cabe incluir en el concepto de motivos imperiosos de seguridad pública que pueden justificar una medida de expulsión”. Pero la individualización del asunto la deja en manos del juez nacional, al igual que la valoración del derecho de protección reforzada, según entienda la importancia de los vínculos del sujeto con el Estado de acogida.

Este pronunciamiento difiere de las Conclusiones del Fiscal General, el cual concluye que el abuso sexual de un menor, la agresión sexual y la violación pueden ser incluidos en el concepto de "motivos imperiosos de seguridad pública". Mientras que, como recogíamos anteriormente, niega el derecho a tal protección reforzada por residencia superior a diez años, debido al uso fraudulento y falta de vínculo y arraigo que implicó su conducta criminal prolongada. 
Revista Internacional de

Doctrina y Jurisprudencia 